\title{
Immediate regraft of the remnant skin on the donor site in split-thickness skin grafting
}

\author{
Young Ji Park, \\ Woo Sang Ryu, \\ Jun Oh Kim, \\ Gyu Hyeon Kwon, \\ Jun Sik Kim, \\ Nam Gyun Kim, \\ Kyung Suk Lee \\ Department of Plastic and \\ Reconstructive Surgery, Gyeongsang \\ National University Hospital, Jinju, \\ Korea
}

\begin{abstract}
Background: Skin defects of head and neck need reconstruction using various local flaps. In some cases, surgeons should consider skin graft for large skin defect. It is important to heal skin graft and donor sites. The authors investigated wound healing mechanisms at the donor sites with split-thick-ness skin graft (STSG). In this study, the authors compared two types of immediate regraft including sheets and islands for the donor site after facial skin graft using remnant skin.

Methods: The author reviewed 10 patients who underwent STSG, from March 2015 to May 2017, for skin defects in the craniofacial area. The donor site was immediately covered with the two types using remnant skin after harvesting skin onto the recipient site. Depending on the size of the remnant skin, we conducted regraft with the single sheet $(n=5)$ and island types $(n=5)$.

Results: On postoperative day 1 and 3 months, the scar formation was evaluated using the Patient and Observer Scar Assessment Scale (POSAS) and Vancouver Scar Scale (VSS). Total POSAS and VSS scores for the island type were lower than in single sheet group after 3 months postoperatively. There was significant difference in specific categories of POSAS and VSS.

Conclusion: This study showed a reduction in scar formation following immediate regrafting of the remnant skin at the donor site after STSG surgery. Particularly, the island type is useful for clinical application to facilitate healing of donor sites with STSG.
\end{abstract}

Keywords: Skin neoplasms / Skin transplantation / Wound healings

\section{INTRODUCTION}

Various methods are available for the reconstruction of head and neck skin defects after wide excision of facial skin cancer, infection or trauma. Minimally invasive surgery such as primary closure and local flap is desirable; however, a skin graft is inevitable if the skin defect is large. The split-thickness skin graft (STSG) surgery has been utilized globally to cover open wounds like skin defects. It is an appropriate method for the reconstruction of skin defects resulting from trauma or burn $[1,2]$. Even though other dressing methods and reconstructive

\section{Correspondence: Kyung Suk Lee}

Department of Plastic and Reconstructive Surgery, Gyeongsang National

University Hospital, 79 Gangnam-ro, Jinju 52727, Korea

E-mail: opensound@hanmail.net

Received March 15, 2019 / Revised April 12, 2019 / Accepted April 15, 2019 operations are available to repair large skin defects, skin graft has been regarded as the first choice or the gold standard treatment to cover large skin defects [3].

STSG for facial skin defects requires postoperative care not only at the recipient but also the donor sites. Often, the treatment duration is extended due to donor site complications even though the graft of the harvested skin is complete. Despite the availability of several dressing methods including moist and dry treatments for the donor site, the optimal dressing methods are disputed [4]. Reported complications at the donor sites include hyperpigmentation (55.4\%), dyschromia (37.5\%), hypertrophic scar (3.6\%), and itching (3.6\%) [5]. The purpose of dressing the donor sites is to facilitate rapid and affordable healing with minimal pain, without infection, and with minimal scarring [6]. Development of appropriate dressing methods continues to be 
the focus of research studies.

Although multiple studies have investigated dressing methods to heal the wounds and leave minimal scars at the donor sites, a proven and universally applicable method has yet to be reported. A recent study reported that an immediate skin graft facilitates epithelization and wound healing along with a reduction in pain [1]. Therefore, we hypothesized that an immediate skin graft diminishes the hypertrophic scar.

This study evaluated donor site complications such as hyperpigmentation, dyschromia and hypertrophic scars by comparing the two groups based on the type of immediate regraft. The size of the skin harvested from the donor site for STSG usually exceeds that of the recipient site. After coverage of the recipient sites the remnant skin is discarded [7]. For the purpose of this study, we introduced two ways for an immediate regraft of the remnant skin, resulting from the STSG in head and neck skin defects, at the donor site and analyzed the donor site scars after the regraft.

\section{METHODS}

\section{Patients}

The present study was conducted in patients who underwent STSG, from March 2015 to May 2017, for facial skin defect due to wide excision of skin cancer or trauma at the Department of Plastic and Reconstructive Surgery. The exclusion criteria were (1) surgical history of the donor site, (2) need for grafting at multiple sites, (3) large surface area of the defect, (4) lack of personal or familial history of keloid formation, (5) peripheral vascular disease, (6) smoking, (7) chronic renal disease, (8) steroid medications, and (9) chronic diseases such as hypertension or diabetes mellitus. Patients ( $\mathrm{n}=10,5$ males and 5 females; mean age, 53 years) manifesting statistically insignificant differences in the covering ratio (regraft surface/defect surface) in the two groups were selected (Table 1).

\section{Surgical procedure}

During the STSG, a plastic surgeon harvested the skin (0.35mm thickness) from the medial thigh using a Zimmer pneumatic dermatome (Zimmer Biomet, Warsaw, IN, USA) and the remnant skin following the skin graft was immediately regrafted onto the donor site. The donor site was immediately covered with the remnant skin after grafting the harvested skin onto the recipient site. Depending on the size of the remnant skin, whether a relatively large single piece or multiple pieces of smaller size, patients were divided into two groups including single sheet $(n=5)$ and island $(n=5)$, respectively (Fig. 1$)$. The margin of the regrafted skin was treated with an anchoring suture, after completion of each regraft, to fix the remnant skin.

\section{Management of the regraft site}

Calcium sodium alginate dressing was used as the standard moist dressing for the donor site in all the subjects [8]. The donor site dressing was changed every day, and healing at the do-

Table 1. Demographics and surgical characteristics of study subjects

\begin{tabular}{|c|c|c|c|c|}
\hline Characteristic & One sheet type group $(n=5)$ & Island type group $(n=5)$ & Total $(n=10)$ & $p$-value ${ }^{a)}$ \\
\hline \multicolumn{5}{|l|}{ Sex } \\
\hline Female & $2(40)$ & $3(60)$ & $5(50)$ & 1.000 \\
\hline Male & $3(60)$ & $2(40)$ & $5(50)$ & 1.000 \\
\hline Age (yr) & $53.0(45.0-66.0)$ & $53.0(14.0-76.0)$ & $53.0(14.0-76.0)$ & 1.000 \\
\hline \multicolumn{5}{|l|}{ Wound location } \\
\hline Scalp & $2(40)$ & $1(20)$ & $3(30)$ & 1.000 \\
\hline Forehead & $2(40)$ & $1(20)$ & $3(30)$ & \\
\hline Cheek & $1(20)$ & $2(40)$ & $3(30)$ & \\
\hline Chin & 0 & $1(20)$ & $1(10)$ & \\
\hline Defect size $\left(\mathrm{cm}^{2}\right)$ & $16.0(12.0-26.5)$ & $9.0(6.0-35.0)$ & $13.5(6.0-35.0)$ & 0.206 \\
\hline \multicolumn{5}{|l|}{ Defect shape } \\
\hline Circular & $1(20)$ & $3(60)$ & $4(40)$ & 0.381 \\
\hline Elliptical & $2(40)$ & $1(20)$ & $3(30)$ & \\
\hline Geographic & 0 & $1(20)$ & $1(10)$ & \\
\hline Irregular & $2(40)$ & 0 & $2(20)$ & \\
\hline Covering ratio ${ }^{\mathrm{b})}$ & $0.28(0.26-0.34)$ & $0.29(0.26-0.37)$ & $0.29(0.26-0.37)$ & 1.000 \\
\hline
\end{tabular}

Values are presented as number (\%) or median (range).

a)The Fisher exact test was conducted for categorical variables (sex, wound location, and defect shape) and the median test for continuous variables (age, defect size, and covering ratio); bovering ratio = regraft surface/defect surface. 

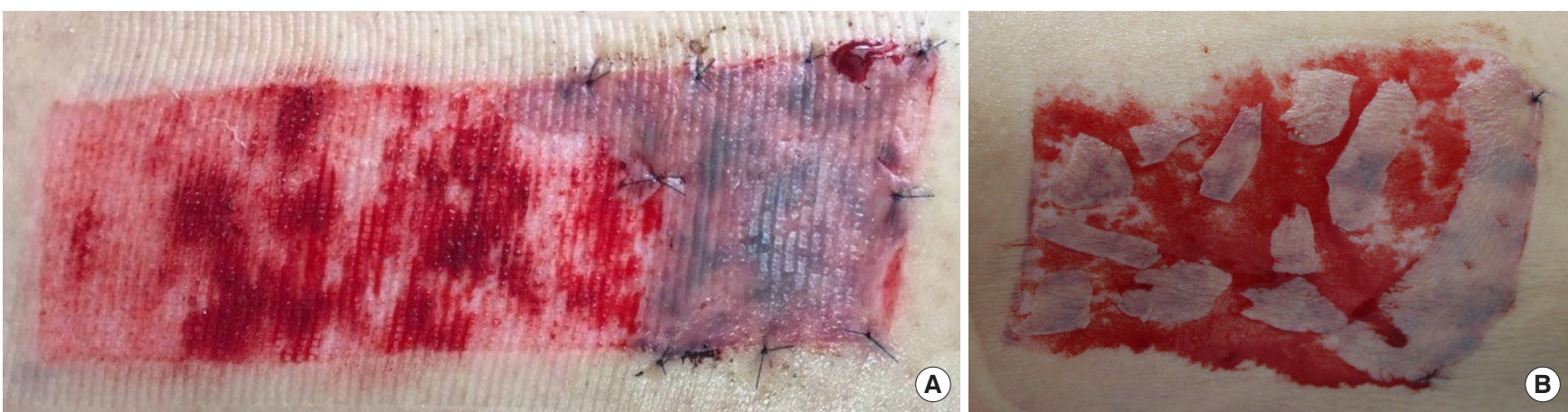

Fig. 1. Photographs of remnant skin immediately regrafted after split-thickness skin graft. (A) Large remnant skin regrafted onto the donor site in the single sheet type. (B) Multiple pieces of the remnant skin regrafted onto randomly selected areas at the donor site in the island type.

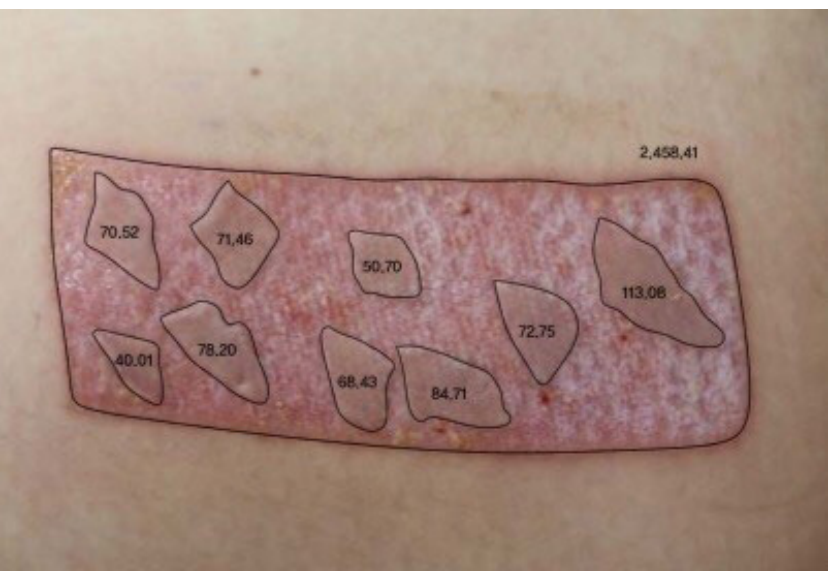

Fig. 2. Computer-aided design was used to calculate the ratio of the regrafted surface area to that of the defect. All data was expressed in millimeters.

nor site was monitored. Next, the gap between the dressing change was extended to 2 days based on the degree of wound oozing. Thereafter, the completed re-epithelialization of the donor site was managed with oil application.

\section{Assessment of the postoperative regraft site}

After regraft at the donor site, independent plastic surgeons (JOK and NGK) conducted a blind test to evaluate scar formation at the donor site based on the Patient and Observer Scar Assessment Scale (POSAS) and Vancouver Scar Scale (VSS).

\section{Measurement of the covering ratio}

To reduce any bias, it was critical to establish the absence of statistically significant difference in the covering ratio between the two groups. To measure the covering ratio, the surface areas of the defect and regraft were measured using computer-aided design technology, and the ratio of the regraft area to that of the defect area was calculated (Fig. 2).

\section{Statistical analysis}

In this study, a nonparametric method was used for statistical analysis for the small-scale study with five subjects per group (10 subjects in total). To compare the demographic and surgical characteristics of single sheet and island types, the Fisher's exact test was performed with discrete variables (sex, wound location, and defect shape), and the median test was performed for continuous variables (age, defect size, and covering ratio) (Table 1). Comparisons between the follow-up times (1 month and 3 months) within a group of the POSAS and VSS were conducted using the Wilcoxon signed-rank test, while the scar scale score of single sheet type and island type was determined using Mann-Whitney $U$ test (Table 2). Intraclass correlation coefficients (ICC) were obtained to verify the reliability of the Observer Scar Assessment Scale and the VSS independently by the two plastic surgeons (Table 3). All statistical analyses were set to two-tailed tests, with a significance level $<0.05$ and performed with SPSS ver. 25.0 (IBM Corp., Armonk, NY, USA).

\section{RESULTS}

Based on the nonparametric method, each variable showed no significant difference in this small but statistically meaningful study (Table 1). We evaluated scar formation in the regraft sites at 1 and 3-months postoperatively using POSAS and VSS (Figs. $3,4)$. Both single sheet and island types showed significant differences in POSAS at 3 months compared with POSAS measured at 1 month postoperatively. Reevaluation of scar formation in each group at 3 months after the procedure revealed statistically significant differences between the two groups, one sheet type and island type, for the mean scores of pigmentation ( $4.3 \pm 0.45$ vs. $2.7 \pm 0.84, p=0.008)$, thickness ( $3.7 \pm 0.57$ vs. $2.9 \pm$ $0.42, p=0.041)$, relief ( $4.1 \pm 0.42$ vs. $2.6 \pm 0.65, p=0.011)$ and pliability ( $4.4 \pm 0.22$ vs. $3.0 \pm 0.50, p=0.007)$ in the Observer Scar Assessment Scale. Additionally, there was a statistically signifi- 
Table 2. Comparison of the POSAS within the groups and between the groups

\begin{tabular}{|c|c|c|c|c|c|c|c|c|c|c|}
\hline \multirow[b]{2}{*}{ Scale } & \multicolumn{4}{|c|}{ One sheet type group $(n=5)$} & \multicolumn{4}{|c|}{ Island type group $(n=5)$} & \multicolumn{2}{|c|}{$p$-value } \\
\hline & 1 Month & 3 Months & $\begin{array}{c}\text { Difference } \\
\text { (1 \& } 3 \text { months) }\end{array}$ & $p$-value $)^{b)}$ & 1 Month & 3 Months & $\begin{array}{c}\text { Difference } \\
\text { (1 \& } 3 \text { months) }\end{array}$ & $p$-value ${ }^{b)}$ & 1 Month & 3 Months \\
\hline \multicolumn{11}{|c|}{ The Patient Scar Assessment Scale } \\
\hline Pain & $5.6 \pm 0.55$ & $2.4 \pm 0.55$ & $3.2 \pm 0.45$ & $0.034^{c)}$ & $5.4 \pm 0.55$ & $2.6 \pm 0.55$ & $2.8 \pm 0.84$ & $0.041^{c)}$ & 0.549 & 0.549 \\
\hline Itching & $5.6 \pm 0.55$ & $2.2 \pm 0.84$ & $3.4 \pm 1.14$ & $0.042^{c)}$ & $5.8 \pm 0.84$ & $2.4 \pm 0.55$ & $3.4 \pm 1.14$ & $0.041^{c)}$ & 0.729 & 0.729 \\
\hline Stiffness & $6.6 \pm 0.55$ & $3.6 \pm 0.55$ & $3.0 \pm 0.71$ & $0.039^{c)}$ & $5.6 \pm 0.55$ & $2.8 \pm 0.84$ & $2.8 \pm 1.10$ & $0.039^{c)}$ & $0.024^{c)}$ & $0.031^{c)}$ \\
\hline Color & $6.8 \pm 0.84$ & $3.2 \pm 0.84$ & $3.6 \pm 0.34$ & $0.041^{c)}$ & $5.8 \pm 0.84$ & $2.6 \pm 0.89$ & $3.2 \pm 0.45$ & $0.034^{c)}$ & $0.035^{c)}$ & 0.268 \\
\hline Thickness & $6.4 \pm 0.55$ & $2.2 \pm 0.84$ & $4.2 \pm 1.10$ & $0.039^{c)}$ & $6.2 \pm 0.84$ & $2.6 \pm 0.89$ & $3.6 \pm 1.14$ & $0.042^{c)}$ & 0.729 & 0.582 \\
\hline Irregularity & $6.0 \pm 0.71$ & $2.6 \pm 0.55$ & $3.4 \pm 0.55$ & $0.038^{c)}$ & $7.4 \pm 0.55$ & $3.8 \pm 0.84$ & $3.6 \pm 1.14$ & $0.042^{c)}$ & $0.016^{c)}$ & $0.033^{c)}$ \\
\hline Total (patient) & $37.0 \pm 1.58$ & $16.2 \pm 1.79$ & $20.8 \pm 3.11$ & $0.043^{c)}$ & $36.2 \pm 1.30$ & $16.8 \pm 1.30$ & $19.4 \pm 1.14$ & $0.042^{c)}$ & 0.393 & 0.735 \\
\hline \multicolumn{11}{|c|}{ The Observer Scar Assessment Scale } \\
\hline Vascularization & $7.9 \pm 0.42$ & $3.4 \pm 0.55$ & $4.5 \pm 0.94$ & $0.042^{c)}$ & $7.5 \pm 0.35$ & $3.1 \pm 0.65$ & $4.4 \pm 0.82$ & $0.042^{c)}$ & 0.142 & 0.347 \\
\hline Pigmentation & $8.0 \pm 0.50$ & $4.3 \pm 0.45$ & $3.7 \pm 0.57$ & $0.034^{c)}$ & $7.2 \pm 0.57$ & $2.7 \pm 0.84$ & $4.5 \pm 0.61$ & $0.041^{c)}$ & 0.055 & $0.008^{c)}$ \\
\hline Thickness & $8.1 \pm 0.42$ & $3.7 \pm 0.57$ & $4.4 \pm 0.42$ & $0.041^{c)}$ & $7.4 \pm 0.55$ & $2.9 \pm 0.42$ & $4.5 \pm 0.50$ & $0.041^{c)}$ & $0.049^{c)}$ & $0.041^{c)}$ \\
\hline Relief & $7.9 \pm 0.42$ & $4.1 \pm 0.42$ & $3.8 \pm 0.84$ & $0.041^{c)}$ & $7.1 \pm 0.55$ & $2.6 \pm 0.65$ & $4.5 \pm 0.61$ & $0.041^{c)}$ & $0.033^{c)}$ & $0.011^{c)}$ \\
\hline Pliability & $7.6 \pm 0.22$ & $4.4 \pm 0.22$ & $3.2 \pm 0.45$ & $0.034^{c)}$ & $7.7 \pm 0.27$ & $3.0 \pm 0.50$ & $4.7 \pm 0.45$ & $0.039^{c)}$ & 0.513 & $0.007^{c)}$ \\
\hline Total & $39.5 \pm 1.06$ & $19.9 \pm 0.82$ & $19.6 \pm 1.71$ & $0.042^{c)}$ & $36.9 \pm 1.34$ & $14.3 \pm 2.51$ & $22.6 \pm 1.56$ & $0.043^{c)}$ & $0.015^{c)}$ & $0.009^{c)}$ \\
\hline Total POSAS & $76.5 \pm 1.46$ & $36.1 \pm 2.16$ & $40.4 \pm 3.27$ & $0.043^{c)}$ & $73.1 \pm 1.75$ & $31.1 \pm 3.34$ & $42.0 \pm 2.15$ & $0.043^{c)}$ & $0.012^{\mathrm{cl}}$ & $0.026^{c)}$ \\
\hline
\end{tabular}

Values are presented as mean \pm standard deviation.

POSAS, Patient and Observer Scar Assessment Scale.

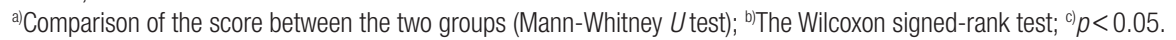

Table 3. Intraclass correlation coefficients for the Observer Scar Assessment Scale and the Vancouver Scar Scale between two plastic surgeons

\begin{tabular}{lll}
\hline \multicolumn{1}{c}{ Scale } & \multicolumn{1}{c}{ ICC $(95 \%$ Cl $)$} & $p$-value \\
\hline $\begin{array}{ll}\text { The Observer Scar Assessment Scale } \\
\text { Vascularization }\end{array}$ & $0.962(0.904$ to 0.985$)$ & $<0.001^{\text {a) }}$ \\
Pigmentation & $0.960(0.898$ to 0.984$)$ & $<0.001^{\text {a) }}$ \\
Thickness & $0.941(0.851$ to 0.977$)$ & $<0.001^{\text {a) }}$ \\
Relief & $0.936(0.839$ to 0.975$)$ & $<0.001^{\text {a) }}$ \\
Pliability & $0.922(0.802$ to 0.969$)$ & $<0.001^{\text {a) }}$ \\
Total & $0.990(0.974$ to 0.996$)$ & $<0.001^{\text {a) }}$ \\
The Vancouver Scar Scale & & \\
Pigmentation & $0.560(-0.112$ to 0.826$)$ & 0.041 \\
Vascularity & $0.694(0.228$ to 0.879$)$ & 0.007 \\
Height & $0.530(-0.187$ to 0.814$)$ & 0.054 \\
Pliability & $0.775(0.432$ to 0.911$)$ & 0.001 \\
Total & $0.889(0.719$ to 0.956$)$ & $<0.001^{\text {a) }}$ \\
\hline
\end{tabular}

ICC, intraclass correlation coefficient; $\mathrm{Cl}$, confidence interval. a) $p<0.001$.

cant difference between the two groups after 3 months, in the mean scores of stiffness ( $3.6 \pm 0.55$ vs. $2.8 \pm 0.84, p=0.031)$ and irregularity ( $2.6 \pm 0.55$ vs. $3.8 \pm 0.84, p=0.033)$ in the patient scar assessment scale. The total POSAS score, obtained after 3 months postoperatively, for the island type $(31.1 \pm 3.34)$ was lower than in the single sheet type $(36.1 \pm 2.16)$ (Table 2$)$.
The total Observer Scar Assessment Scale and the average ICC of total VSSs were 0.990 (95\% confidence interval [CI], 0.974 to $0.996 ; p<0.001$ ) and 0.889 ( $95 \%$ CI, 0.719 to $0.956 ; p<$ $0.001)$, respectively. The assessment of ICC by the two plastic surgeons showed a consistency exceeding $80 \%$, suggesting high reliability (Table 3).

A total of 10 patients were selected to evaluate the scar formation using the VSS, after 1 and 3 months postoperatively (Table 4). Both single sheet and island types manifested significant differences in VSS at 3 months compared with VSS measured at 1 month postoperatively, except for height. Measurements of VSS obtained 1 month after the surgery revealed no statistically significant differences. Interestingly, the evaluation of mean VSS scores, 3 months after completion of the surgery, revealed significant differences between the single sheet and island types in pigmentation $(1.8 \pm 0.27$ vs. $1.2 \pm 0.27, p=0.020)$ and pliability $(2.3 \pm 0.45$ vs. $1.3 \pm 0.27, p=0.014)$. Total VSS scores, determined 1 month after the surgery, in the single sheet and island types were $11.3 \pm 0.76$ and $10.5 \pm 0.71$, respectively. Similarly, VSS scores, determined 3 months after the surgery, in the single sheet and island types were $6.7 \pm 0.27$ and $5.0 \pm 0.79$, respectively.

One patient from the island type group was selected for longterm monitoring of postoperative progress, 6 months after the graft procedure, and the results showed excellent aesthetic outcome at the donor site (Fig. 5). 

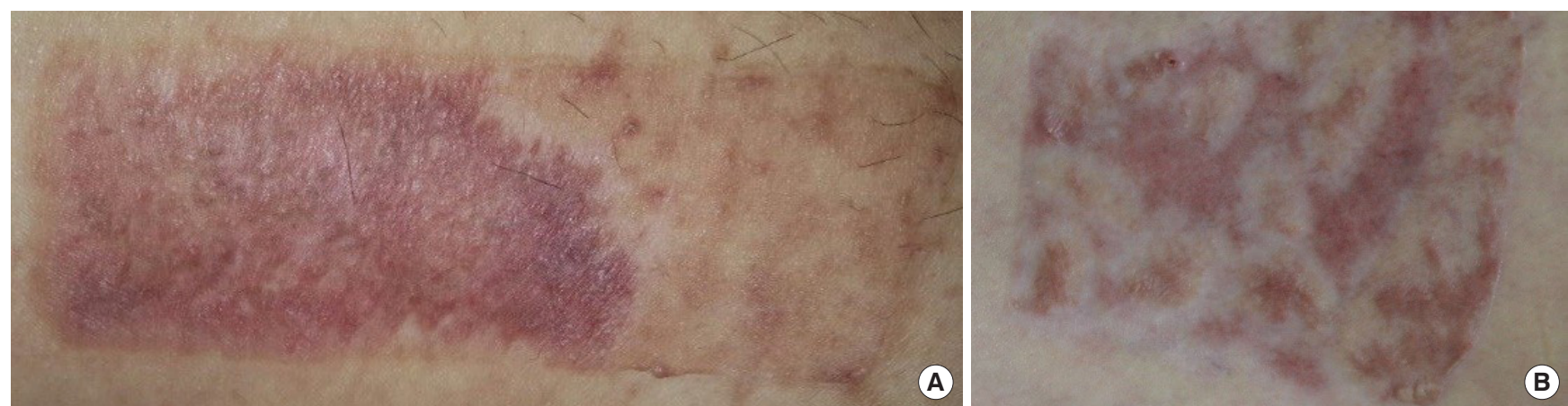

Fig. 3. Photographs of scar formation for each group 1 month after split-thickness skin graft. (A) Single sheet type and (B) island type.
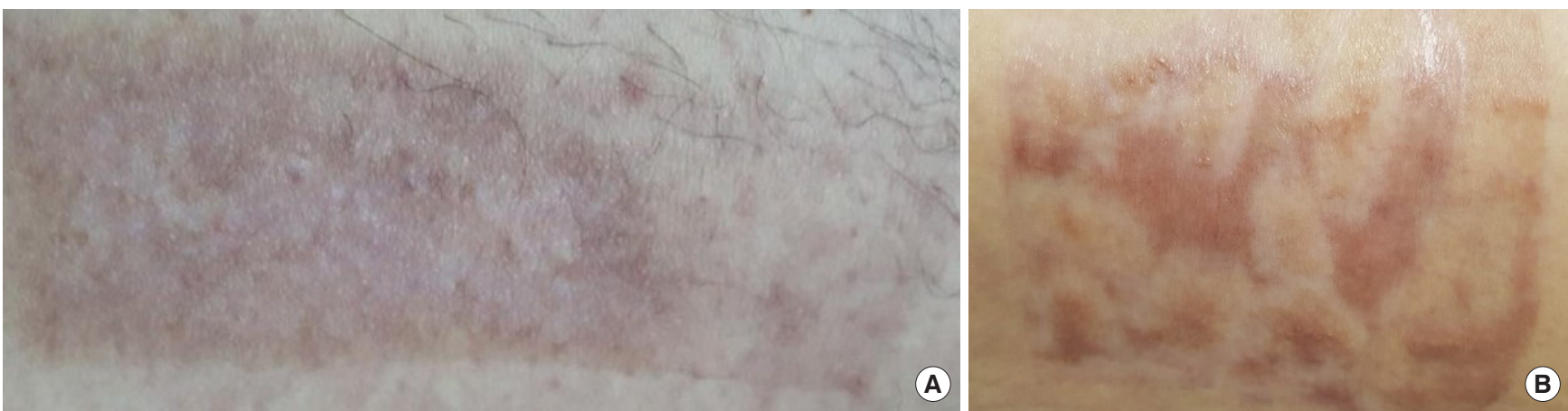

Fig. 4. Scar formation in each group 3 months after split-thickness skin graft. (A) Single sheet and (B) island types.

Table 4. Comparison of the Vancouver Scar Scale within the groups and between the groups

\begin{tabular}{|c|c|c|c|c|c|c|c|c|c|c|}
\hline & \multicolumn{4}{|c|}{ One sheet type group $(n=5)$} & \multicolumn{4}{|c|}{ Island type group $(n=5)$} & \multicolumn{2}{|c|}{$p$-value } \\
\hline & 1 Month & 3 Months & $\begin{array}{c}\text { Difference } \\
\text { (1 \& } 3 \text { months) }\end{array}$ & $p$-value ${ }^{b)}$ & 1 Month & 3 Months & $\begin{array}{c}\text { Difference } \\
\text { (1 \& } 3 \text { months) }\end{array}$ & $p$-value ${ }^{b)}$ & 1 Month & 3 Months \\
\hline \multicolumn{11}{|c|}{ The Vancouver Scar Scale } \\
\hline Pigmentation & $2.7 \pm 0.27$ & $1.8 \pm 0.27$ & $0.9 \pm 0.42$ & $0.041^{c)}$ & $2.3 \pm 0.27$ & $1.2 \pm 0.27$ & $1.1 \pm 0.42$ & $0.041^{c)}$ & 0.058 & $0.020^{c)}$ \\
\hline Vascularity & $2.4 \pm 0.22$ & $0.9 \pm 0.55$ & $1.5 \pm 0.50$ & $0.041^{c)}$ & $2.5 \pm 0.00$ & $1.2 \pm 0.67$ & $1.3 \pm 0.67$ & $0.041^{c)}$ & 0.317 & 0.419 \\
\hline Height & $2.6 \pm 0.22$ & $1.7 \pm 0.45$ & $0.9 \pm 0.55$ & 0.059 & $2.2 \pm 0.27$ & $1.3 \pm 0.57$ & $0.9 \pm 0.65$ & 0.066 & 0.052 & 0.290 \\
\hline Pliability & $3.6 \pm 0.55$ & $2.3 \pm 0.45$ & $1.3 \pm 0.97$ & $0.039^{c)}$ & $3.5 \pm 0.35$ & $1.3 \pm 0.27$ & $2.2 \pm 0.45$ & $0.039^{c)}$ & 0.906 & $0.014^{c)}$ \\
\hline Total & $11.3 \pm 0.76$ & $6.7 \pm 0.27$ & $4.6 \pm 0.65$ & $0.043^{c)}$ & $10.5 \pm 0.71$ & $5.0 \pm 0.79$ & $5.5 \pm 0.94$ & $0.043^{\mathrm{c})}$ & 0.147 & $0.008^{c)}$ \\
\hline
\end{tabular}

Values are presented as mean \pm standard deviation.

${ }^{a}$ Comparison of the score between the two groups (Mann-Whitney U test); ${ }^{b)}$ Wilcoxon signed-rank test; ${ }^{c} p<0.05$.

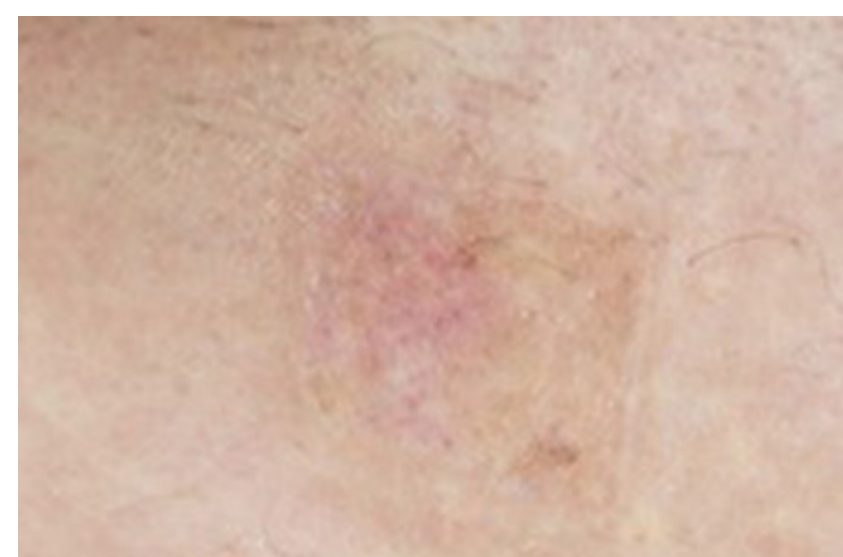

Fig. 5. An island type donor site 6 months after the graft procedure.

\section{DISCUSSION}

In the present study, two independent plastic surgeons and patients evaluated scar formation using POSAS and VSS, 1 and 3 months after the STSG procedure. Measurements of total POSAS, obtained 1 and 3 months after STSG, revealed a statistically significant reduction in the island type compared with the single sheet type. Additionally, the measurements of Observer Scar Assessment Scale 3 months after the STSG procedure showed a statistically significant reduction in pigmentation, thickness, relief, and pliability of scar formation.

Previous studies demonstrated positive wound healing effects at the donor sites when regrafting was performed immediately 
using remnant skin during the STSG operation [9]. In cases of skin harvested with a dermatome, most of the harvested skin is usually rectangular in shape. However, remnant skins in different shapes inevitably result from the skin graft due to the diverse shapes of the recipient sites. In this study, we hypothesized that immediate regrafting of the remnant skin following STSG at the donor site leads to positive effects on scar formation.

Based on the POSAS scores, we found that the patient's symptoms improved during the 3 months after the procedure. POSAS scores obtained 1 month after the procedure revealed a statistically significant decrease in stiffness and color in the island type compared with the single sheet group. Moreover, POSAS scores evaluated 3 months after the procedure revealed a statistically significant decrease in stiffness of patients in the island type when compared with the single sheet group.

Interestingly, compared with the patient scar assessment scale, the irregularity score in the island type at both the time-points postoperatively, suggested a lower level of satisfaction. This outcome may be attributed to the irregularities in size and the interval between the regrafting site of the remnant skins in the island type group. Interestingly, the island type group showed a better aesthetic appearance than the single sheet type. By contrast, the total POSAS score revealed a higher score in the single sheet group compared with the island type group.

Further, the comparison of the VSS scores obtained 1 month after STSG did not reveal any statistically significant differences between the two groups. Interestingly, the scores obtained 3 months after the procedure showed statistically significant decreases in pigmentation and pliability of the island type compared with the single sheet type.

Based on POSAS and VSS, this study found reduced scar formation with the island type regraft compared with the single sheet. An objective study of the regraft-induced scar formation was facilitated by excluding the positive effects due to the significant differences in the covering ratios of each regraft type, which was achieved by controlling the covering ratios for each regraft type resulting in statistically insignificant differences.

The island type regraft at the donor site may exert additional molecular effects on the adjacent raw surface compared with the single sheet due to the relatively larger surface area of epithelization. Using the same covering ratios in each type, statistically insignificant differences were detected. An increased covering ratio due to an enlarged area of the regrafted skin as a result of epithelization was observed in the island type regraft.

We used various statistical methods to analyze insufficient number of cases to validate significant results. We found significant differences with various statistical methods to compensate for the small number of cases; however, results based on addi- tional cases will enhance the reliability.

The study limitations relate to insufficient number of cases, short follow-up duration, and lack of diversity in donor sites for skin harvest. Additionally, the interval between each island type regraft was inconsistent due to the immediate regraft of the remnant skin at the donor sites. Future studies should focus on the determination of regraft intervals from the diverse donor sites accompanied by experimental evaluation of molecular factors underlying the reduction in scar formation.

The present study identified the reduction in scar formation following immediate regrafting of the remnant skin at the donor site after the STSG surgery. Particularly, compared with the single sheet, the regraft using the island type remnant skin reduced scar formation at the donor site. Therefore, an immediate regraft of the remnant skin (island type) at the donor site is expected to be effective clinically.

\section{NOTES}

\section{Conflict of interest}

No potential conflict of interest relevant to this article was reported.

\section{Ethical approval}

The study was performed in accordance with the principles of the Declaration of Helsinki. Written informed consents were obtained from patients.

\section{Patient consent}

The patients provided written informed consent for the publication and the use of their images.

\section{ORCID}

$\begin{array}{ll}\text { Young Ji Park } & \text { https://orcid.org/0000-0001-8416-7833 } \\ \text { Woo Sang Ryu } & \text { https://orcid.org/0000-0002-7472-3813 } \\ \text { Jun Oh Kim } & \text { https://orcid.org/0000-0002-3860-6433 } \\ \text { Gyu Hyeon Kwon } & \text { https://orcid.org/0000-0002-8377-4698 } \\ \text { Jun Sik Kim } & \text { https://orcid.org/0000-0001-7271-3868 } \\ \text { Nam Gyun Kim } & \text { https://orcid.org/0000-0002-1511-9106 } \\ \text { Kyung Suk Lee } & \text { https://orcid.org/0000-0001-7731-1761 }\end{array}$

\section{REFERENCES}

1. Bian Y, Sun C, Zhang X, Li Y, Li W, Lv X, et al. Wound-healing improvement by resurfacing split-thickness skin donor sites with thin split-thickness grafting. Burns 2016;42:123-30.

2. Dornseifer U, Lonic D, Gerstung TI, Herter F, Fichter AM, Holm C, et al. The ideal split-thickness skin graft donor-site 
dressing: a clinical comparative trial of a modified polyurethane dressing and aquacel. Plast Reconstr Surg 2011;128:91824.

3. Anderson JJ, Wallin KJ, Spencer L. Split thickness skin grafts for the treatment of non-healing foot and leg ulcers in patients with diabetes: a retrospective review. Diabet Foot Ankle 2012; 3:10204.

4. Voineskos SH, Ayeni OA, McKnight L, Thoma A. Systematic review of skin graft donor-site dressings. Plast Reconstr Surg 2009;124:298-306.

5. Otene CI, Olaitan PB, Ogbonnaya IS, Nnabuko RE. Donor site morbidity following harvest of split-thickness skin grafts in South Eastern Nigeria. J West Afr Coll Surg 2011;1:86-96.
6. Innes ME, Umraw N, Fish JS, Gomez M, Cartotto RC. The use of silver coated dressings on donor site wounds: a prospective, controlled matched pair study. Burns 2001;27:621-7.

7. Knapik A, Kornmann K, Kerl K, Calcagni M, Contaldo C, Vollmar B, et al. Practice of split-thickness skin graft storage and histological assessment of tissue quality. J Plast Reconstr Aesthet Surg 2013;66:827-34.

8. Barrit DP, Brike-Sorensen H. Dressing for split thickness skin graft donor sites: a comparison of three options. EWMA J 2014;14:15-20.

9. Bradow BP, Hallock GG, Wilcock SP. Immediate regrafting of the split thickness skin graft donor site assists healing. Plast Reconstr Surg Glob Open 2017;5:e1339. 